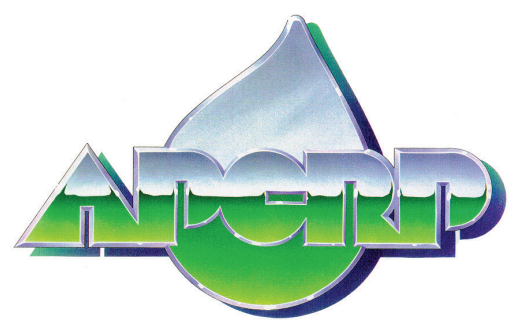

ERDC/TN APCRP-BC-39

September 2017

\title{
Surveys for Biological Control Agents of Hydrilla verticillata and Myriophyllum spicatum in the Republic of Korea and the People's Republic of China in 2014
}

\author{
by Matthew Purcell', Nathan E. Harms ${ }^{2}$, Michael J. Grodowitz ${ }^{2}$, \\ Jialiang Zhang ${ }^{3}$, Myung-Hyuun $\mathrm{Kim}^{4}$, Kwang-Jin $\mathrm{Cho}^{4}$, \\ Young J. Oh ${ }^{5}$, Sun-Hee Hong ${ }^{6}$, and Jianqing Ding ${ }^{3}$
}

PURPOSE: The purpose of this Technical Note (TN) is to summarize overseas work conducted in 2014 to locate insect biological control agents of dioecious and monoecious Hydrilla verticillata (L.f.) Royle (hydrilla) and Myriophyllum spicatum L. (Eurasian Water Milfoil) (EWM) in the Republic of Korea (RK) and the People's Republic of China (PRC). This work includes surveys of hydrilla and EWM sites in the RK and the PRC, and represents ongoing efforts towards identifying previously collected and reared agents.

INTRODUCTION: Hydrilla is a submersed invasive plant that is native to Africa, Asia, Australia, and parts of Europe; it has been present in the United States since the 1950s (Buckingham and Grodowitz 2004; Schmitz et al. 1991). Two genetically and ecologically distinct hydrilla biotypes are currently recognized in the U.S.; a pistillate dioecious biotype which is generally found in the southeastern and south central region and a monoecious biotype which mostly occurs in the central Atlantic and northeastern regions. (Madeira et al. 2000). Though the dioecious biotype has been present in the U.S. for over sixty years, monoecious hydrilla is widely recognized as a more recent introduction. Monoecious hydrilla was first discovered in the northeastern region of the U.S. in 1976 (Steward et al. 1984) and since then it has been reported in Alabama, California, Connecticut, Delaware, Georgia, Indiana, Kansas, Kentucky, Maine, Massachusetts, Maryland, Missouri, North Carolina, New Jersey, New York, Ohio, Pennsylvania, South Carolina, Tennessee, Virginia, Washington, West Virginia, and Wisconsin (Jacono 2017).

\footnotetext{
${ }^{1}$ USDA-ARS, Australian Biological Control Laboratory (ABCL), 41 Boggo Road, Dutton Park, Queensland, Australia 4102. Phone 617-38335740; Fax 617-38335504; Email Matthew.Purcell@,csiro.au.

${ }^{2}$ U.S. Army Engineer Research and Development Center, Environmental Laboratory, Aquatic Ecology and Invasive Species Branch, 3909 Halls Ferry Rd., Vicksburg, MS 39180, USA.

${ }^{3}$ Invasion Biology and Biocontrol Lab, Wuhan Botanical Institute, Chinese Academy of Sciences, Wuhan, Hubei Province, 430074 China. Email: ding@wbgcas.cn.

${ }^{4}$ Rural Development Administration, National Academy of Agricultural Science, 126 Suin-Ro, Kweonseonku, Suwon 441-707, Korea. Email: wildflower72@korea.kr.

${ }^{5}$ Institute for Future Environmental Ecology Co., Ltd., 441-853 Seoho-ro, 89th, Gwonseon-gu, Suwon, Gyeonggi-go, Seoul National University, Bio Venture Valley 1-308. Email: cave50joo@gmail.com.

${ }^{6}$ Institute of Environment and Ecology, Division of Environmental Science and Ecological Engineering, College of Life Science and Biotechnology, Anam-dong, Seongbuk-gu, Seoul 136-713 Korea. Email: cootation@korea.ac.kr.
} 
Hydrilla (biotypes not reported) have been surveyed for biological control agents in various parts of its native range since the 1960s (Balciunas et al. 2002) which led to the testing and release of two ephydrid flies (Hydrellia pakistanae Deonier and H. balciunasi Bock) and two curculionid beetles (Bagous hydrillae O'Brien and B. affinis Hustache) in the U.S. in the late 1980s and early 1990s. Only $H$. pakistanae and $H$. balciunasi established and dispersed, principally on dioecious hydrilla populations in the southern U.S., with $H$. pakistanae being the most widespread and commonly encountered agent (Grodowitz et al. 2004).

Monoecious hydrilla has been reported in 23 states within the U.S. as it rapidly spreads into a large variety of waterways (Grodowitz et al. 2010; Jacono 2017). While research has demonstrated that the introduced $H$. pakistanae flies impact the monoecious biotype under laboratory conditions, more recent evidence suggests that biotype-specific differences in the plant's overwintering and canopy formation will not allow the agents to establish over the long term (Dray and Center 1996; Grodowitz et al. 2010; Harms and Grodowitz 2011). Because the origins of the hydrilla biotype (monoecious/ dioecious) from which these insects were collected in the native range is unknown, surveys for biological control agents that target both dioecious and monoecious biotypes in their native ranges commenced in the PRC in $2013^{1}$. This approach was deemed to be the most efficient means for identifying and testing agents that are best suited for either biotype (Harms et al. 2014).

Indigenous to Europe, Asia, and North Africa, and considered a serious waterweed in regions of the U.S. is the EWM (Couch and Nelson 1985). Millions of dollars are spent annually on the control of EWM and it is considered one of the most important introduced aquatic weeds in within the continental U.S. (U.S. Congress, Office of Technology Assessment, 1993). The introduction of EWM negatively affects wildlife and fish populations, decreases native macrophyte abundance and diversity, hinders or prevents recreational water use, disrupts water flow, and increases siltation (Buckingham 2004; Johnson and Blossey 2002; Madsen et al. 1991; Smith and Barko 1990). Currently, EWM can be found from Alaska to Florida and occurs in most U.S. states and three Canadian provinces (Buckingham 2004).

Neither the geographic center of EWM evolution nor the geographic origins of its introductions into the U.S. are known. Native to Eurasia and North Africa, EWM surveys for biological control agents have been conducted in Europe and Asia (Eiswerth et al. 2000; Johnson and Blossey 2002). Despite more than twenty insects identified as attacking EWM in its native range, no biological control agents have been deliberately imported into the U.S. for control. However, the generalist aquatic moth Acentria ephemerella Denis and Schiffermüller (Lepidoptera: Pyralidae) is native to Europe and appeared in North America in 1927 (Sheppard 1945). This moth is now widespread and has been associated with declines of EWM populations in some areas (Painter and McCabe 1988; Johnson et al. 1997; Gross et al. 2001). Two native Curculionidae, Euhrychiopsis lecontei Dietz and Litodactylus leucogaster Marsham and a native midge, Cricoptopus myriophylli Oliver, also feed on EWM. Of these species only E. lecontei has been associated with declines of this weed (Buckingham 2004). Given that EWM continues to spread posing a significant threat to U.S. waterways, the development of new control measures are essential for its management.

\footnotetext{
${ }^{1}$ Harms, N. E., M. Purcell, J. Zhang, M. J. Grodowitz and J. Ding. In Prep. Surveys for Biological Control Agents of Hydrilla verticillata in the People's Republic of China during 2013.
} 
This TN details the expansion of 2013 surveys for biological control agents of monoecious and dioecious hydrilla into the RK and new areas of the PRC during July and August 2014. Given that the general consensus is that the U.S. monoecious hydrilla is genetically most similar to Korean accessions, the continuation of surveys following those conducted in the northern PRC in 2013 were largely focused in the RK. (Harms et al. 2014; Madeira et al. 2007; Benoit 2011) Additionally, given that previous RK surveys were limited geographically, more widespread examination was warranted. Herbivorous insects attacking EWM were also evaluated when this plant was encountered. Some sites surveyed in the PRC in 2013 were also revisited.

\section{MATERIALS AND METHODS}

Site Parameters. The following environmental conditions were recorded at sites where hydrilla or EWM were collected: water temperature, $\mathrm{pH}$, salinity and total dissolved solids measured using an ECPCSTestr 35 meter as well as Secchi depth, and water depth. Categorical site characteristics were also recorded: human presence (low/medium/high), sediment type (sand/clay/gravel/shale/sandstone/ muck/rock) and texture (fine/course/heavy/hardpan), water flow (stagnant/flowing) and type of waterbody (man-made/artificial). These data were used for correlating the presence of the hydrilla or EWM in waterbodies and possibly the abundance of herbivore species in future surveys.

Surveys for Herbivores. Sites where hydrilla and EWM occurred in the RK were identified through examination of herbarium specimens, online database searches (www.GBIF.org), and contacts with the RK scientists. Other site visits in the RK and new sites visited in the provinces of Shanghai, Zhejiang, Jiangsu, Shandong and Hubei in the PRC were facilitated by searching likely waterways on planned road routes. If aquatic vegetation was observed, then the site was examined for the presence of hydrilla and EWM.

The surveys covered a wide array of the aquatic systems of the RK and it is believed that it was a representative sample of hydrilla and EWM within that country. Given the previous extensive exploration conducted in the PRC, the 2014 surveys focused on the poorly surveyed areas from approximately $300 \mathrm{~km}$ south west of Shanghai, north of Beijing. A total of 102 sites were visited, 74 in the RK (17 July-7 August, Figure 1) and 28 in the PRC (10-18 August, Figure 2). As in previous surveys, site types varied and included urban and rural, natural and manmade, ponds, lakes, rivers, manmade canals, shallow irrigation ditches, and natural wetlands. Plants were collected from shore by tossing a double-sided metal rake with a rope handle or by wading and harvesting by hand. Plants were visually examined in the field for 10-30 minutes for signs of herbivory with the aid of a jeweler's loupe (10X magnification). Approximately $1 \mathrm{~kg}$ fresh weight of plant material was collected from each site and sealed in a zip lock plastic bag. When present, the sex of flowers on hydrilla was recorded and the biotype (monoecious/dioecious) was estimated. 


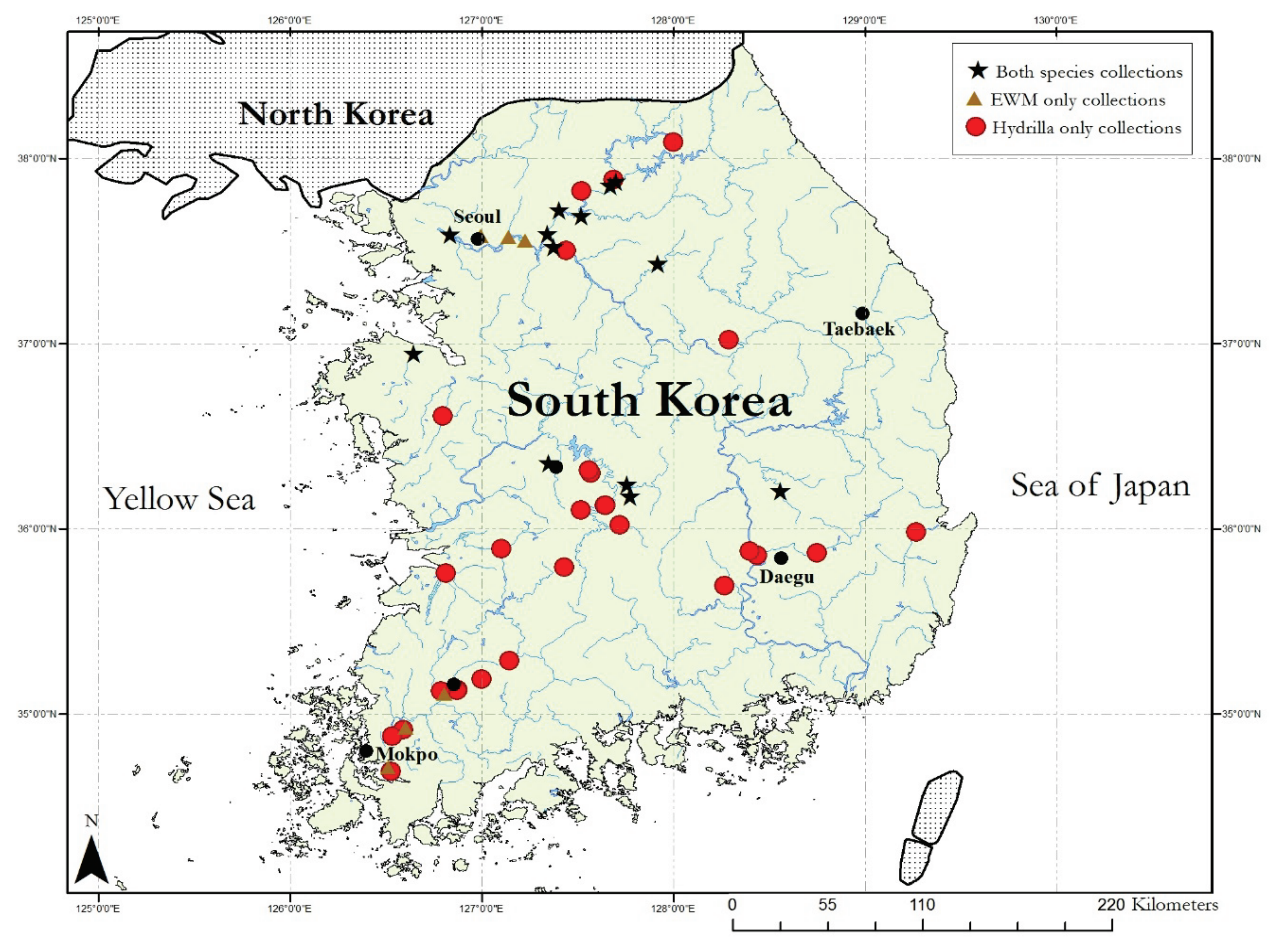

Figure 1. 2014 hydrilla and EWM herbivore sampling locations in the RK.

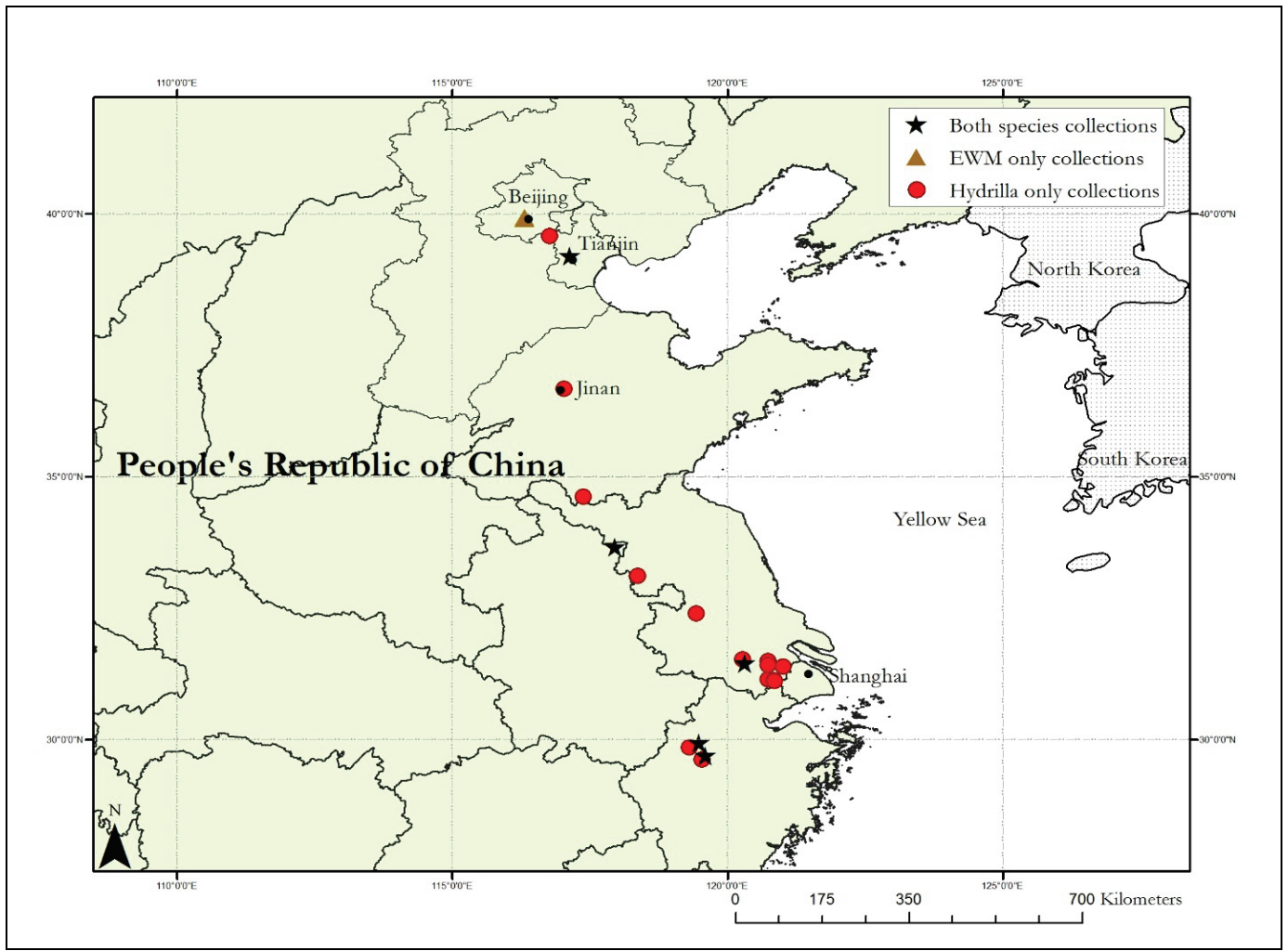

Figure 2. 2014 hydrilla and EWM herbivore sampling locations in the PRC. 
Processing Samples, Identification and Rearing of Herbivore Species. Plant samples were processed in Berlese funnels in hotel rooms where refrigeration was usually available to prevent degradation of stored plant material. Portable, cloth Berlese funnels (Bioquip, Rancho Dominguez, CA) utilizing a single 40 watt incandescent light bulb were used to progressively dry the hydrilla and EWM material and drive internally and externally feeding herbivores into a collection vessel containing water. These were inspected daily until the plant material in the Berlese was dry and the herbivores were extracted. Using a fine camel hair brush, aquatic pyralid moth larvae and Hydrellia spp. immatures were carefully transferred into rearing containers (small variously-sized plastic containers and $30 \mathrm{~mm} \times 55 \mathrm{~mm}$ diameter capped plastic tubes containing $\sim 5 \mathrm{~cm}$ sprig of hydrilla from the site it was collected from and $\sim 1 \mathrm{~cm}$ of water) in an effort to rear them to adulthood, verify identifications, as well as document feeding damage. Chironomidae larvae were preserved in $95 \%$ $\mathrm{EtOH}$ as they are difficult to rear without aeration of the water within the rearing cups. However, some were monitored in rearing containers to identify feeding damage. Adults and immatures of other herbivores were preserved in $95 \% \mathrm{EtOH}$ as voucher specimens for later identification.

\section{RESULTS AND DISCUSSION}

Site Conditions. Water quality and characteristics of hydrilla and EWM collection sites are provided in Tables 1 and 2. Observations worth contrasting between the RK and the PRC are salinity and total dissolved solids which were both substantially higher in the PRC. This was possibly due to higher levels of contaminants in the waterways examined in the PRC. Hydrilla (and to some extent EWM) were identified in a wide range of water qualities and physical parameters. For example, plants were found at sites with salinities ranging from $30.44 \mathrm{ppm}$ to $798 \mathrm{ppm}$ and $\mathrm{pH}$ from 6.6 to 10.3 .

It was rare to find hydrilla and/or EWM growing in monoculture. Typically, these species were found associated with an assemblage of floating, emergent, and submersed plant species, unlike in the U.S. where these species often crowd out other species. The mean number of taxa found at hydrilla and/or EWM sites was 4.4 with a maximum of 17 different plant taxa identified at one site. Mean number of taxa were similar for the RK and the PRC with means of 4.2 and 4.9, respectively. The most commonly encountered genus was Ceratophyllum in 30 sites followed by Lemna in 24 sites, Trapa in 23 sites and Potamogeton in 21 sites. Other common genera identified included Najas, Nymphoides, and Nymphaea.

Table 1. Water quality and physical parameters measured at hydrilla and EWM collection sites in the RK and PRC in 2014.

\begin{tabular}{||l|c|c|c|c||c|c|c|c||}
\hline \multirow{2}{*}{\multicolumn{1}{c|}{ Parameter }} & \multicolumn{4}{c||}{ Republic of Korea } & \multicolumn{3}{c||}{ Peoples Republic of China } \\
\cline { 2 - 11 } & Mean (n) & Min & Max & SE & Mean (n) & Min & Max & SE \\
\hline \hline Temperature ( $(\mathrm{C})$ & $27.0(48)$ & 21.6 & 33.3 & 0.39 & $27.6(20)$ & 23.7 & 31.6 & 0.44 \\
\hline pH & $8.2(48)$ & 6.6 & 10.2 & 0.12 & $8.4(20)$ & 7.4 & 10.3 & 0.17 \\
\hline Salinity (ppm) & $128.5(46)$ & 30.4 & 571.0 & 14.2 & $255.5(20)$ & 56.0 & 798.0 & 38.6 \\
\hline $\begin{array}{l}\text { Total Dissolved Solids } \\
\text { (ppm) }\end{array}$ & $199.8(46)$ & 40 & 891 & 22.6 & $401.7(20)$ & 79 & 1210 & 59.9 \\
\hline Secchi Depth (cm) & $34.6(48)$ & 1 & 100 & 2.9 & $38.0(20)$ & 15 & 60 & 3.4 \\
\hline Water Depth (cm) & $50.8(48)$ & 1 & 160 & 5.7 & $74.0(20)$ & 15 & 250 & 13.9 \\
\hline
\end{tabular}




\begin{tabular}{|c|c|c|c|}
\hline & & & \\
\hline & & & \\
\hline & & Korea & China \\
\hline & Low & $15.9 \%(7)$ & $15.0 \%(3)$ \\
\hline Human Presence ${ }^{1}$ & Medium & $61.4 \%(27)$ & $30.0 \%(6)$ \\
\hline & High & $22.7 \%(19)$ & $55.0 \%(11)$ \\
\hline & Fine & $68.2 \%(30)$ & $100.0 \%(20)$ \\
\hline Sediment Tyne2 & Course & $25.0 \%(11)$ & $0.0 \%(0)$ \\
\hline Sedıment Iуре & Muck & $2.3 \%(1)$ & $0.0 \%(0)$ \\
\hline & Rock & $0.0 \%(0)$ & $0.0 \%(0)$ \\
\hline Whton $\Gamma$ Ta & Stagnant & $40.9 \%(18)$ & $50.0 \%(10)$ \\
\hline Water Flow & Flowing & $59.1 \%(26)$ & $50.0 \%(10)$ \\
\hline Tyne of W/aterhodv & Man-made/ modified & $63.4 \%(28)$ & $55.0 \%(11)$ \\
\hline Type of Vaterbody & Natural & $36.4 \%(16)$ & $45.0 \%(9)$ \\
\hline & Eurasiar & rmilfoil & \\
\hline & & & \\
\hline & & Korea & China \\
\hline & Low & $21.1 \%(4)$ & $14.3 \%(1)$ \\
\hline Human Presence ${ }^{1}$ & Medium & $57.9 \%(11)$ & $28.6 \%(2)$ \\
\hline & High & $21.1 \%(4)$ & $57.1 \%(4)$ \\
\hline & Fine & $68.4 \%(13)$ & $100.0 \%(7)$ \\
\hline Codimont Tinn? & Course & $26.3 \%(5)$ & $0.0 \%(0)$ \\
\hline Sedıment Iypez & Muck & $0.0 \%(0)$ & $0.0 \%(0)$ \\
\hline & Rock & $5.3 \%(1)$ & $0.0 \%(0)$ \\
\hline Water Flow & Stagnant & $42.1 \%(8)$ & $57.1 \%(4)$ \\
\hline Wvater Flow & Flowing & $57.9 \%(11)$ & $42.9 \%(3)$ \\
\hline Tyno of W/atorhodv & Man-made/ modified & $47.4 \%(9)$ & $42.9 \%(3)$ \\
\hline Iype of Waterbody & Natural & $52.6 \%(10)$ & $57.1 \%(4)$ \\
\hline TOTALS & & $68.8 \%(44)$ & $31.3 \%(20)$ \\
\hline
\end{tabular}

${ }^{1}$ Human Presence - High (water is accessible by large numbers of people - parks, small city ponds, active fishing, etc.); Medium (less access by people but still reachable - small water bodies in smaller towns and villages); Low (natural area, limited use by local population).

${ }^{2}$ Sediment types: Fine: soft clay to sandy-clay mix with some organics present; Coarse: heavy sand to gravel; Muck: unconsolidated with high organic content; Rock: hardpan clay to shale, sandstone, or other rock materials.

Surveys for Herbivore Species. Hydrilla was found at 59\% and EWM at $19 \%$ of the 74 sites examined in the RK. In the PRC, hydrilla was found at 68\% and EWM at 25\% of 28 sites. Both species were collected from $24 \%$ (RK) and 22\% (PRC) of the sites, and neither species was collected from $34 \%(\mathrm{RK})$ and $25 \%$ (PRC) of the sites. As in previous surveys, the reasons for the presence or absence of plants was unclear, unless the aquatic systems were flooded or had been flushed, though it possibly related to aspects such as water quality, substrate and flow rate.

Identification and Rearing of Herbivore Species. Table 3 details sites where hydrilla and EWM were surveyed in 2014 in the RK and the PRC. Also listed are the herbivores collected at each site. 


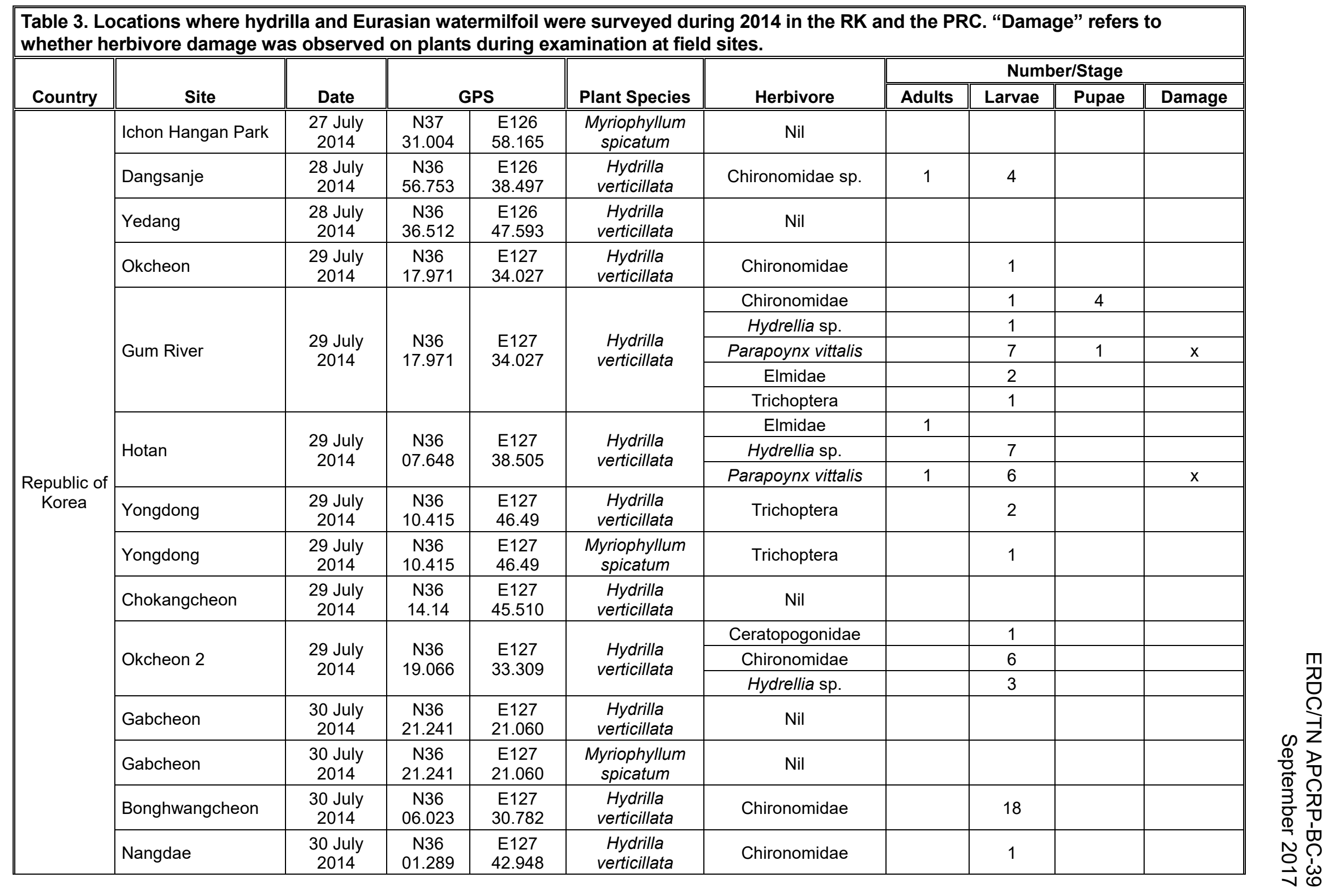




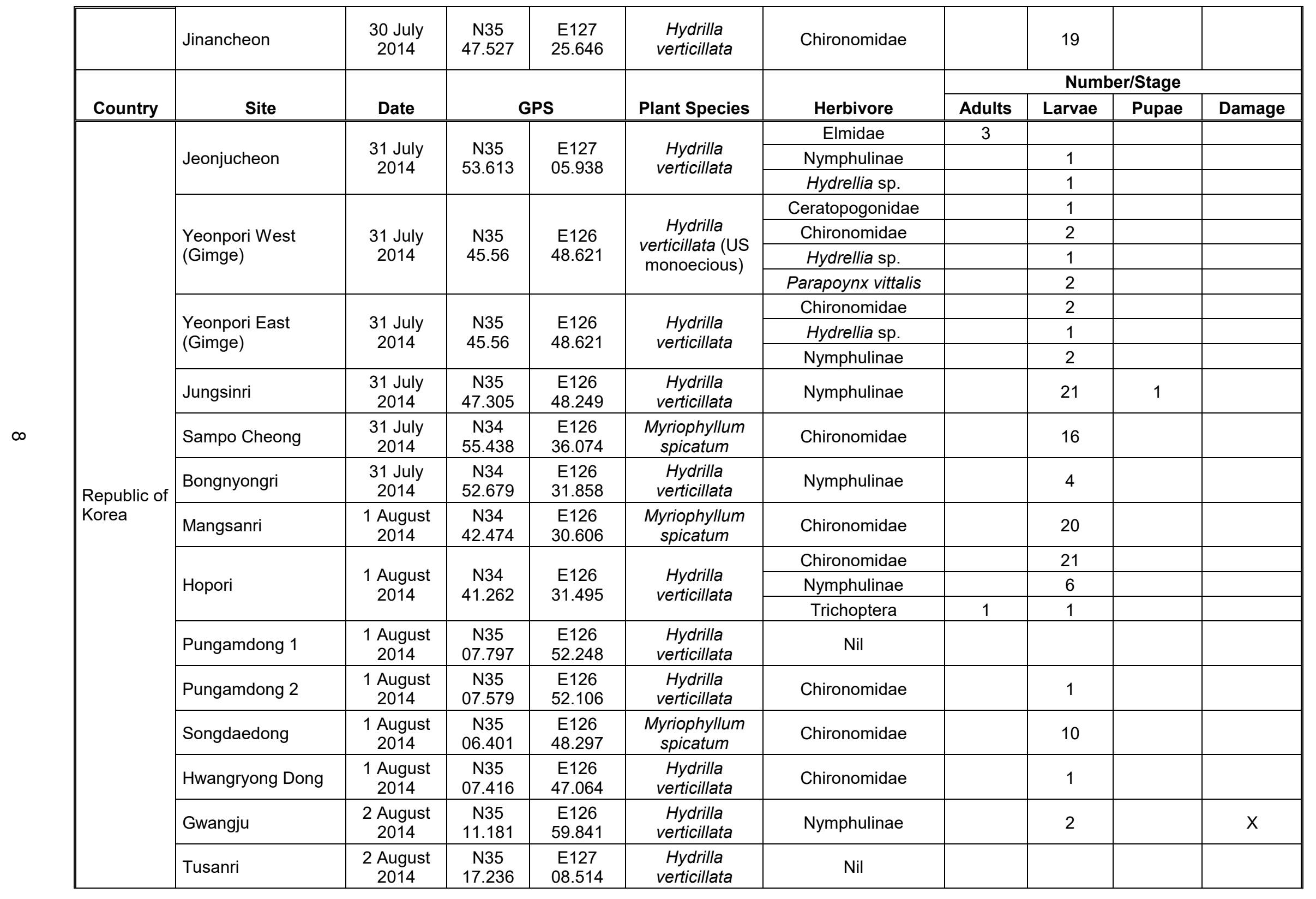




\begin{tabular}{|c|c|c|c|c|c|c|c|c|c|c|}
\hline & \multirow{2}{*}{ Allimri } & \multirow{2}{*}{$\begin{array}{l}2 \text { August } \\
2014\end{array}$} & \multirow{2}{*}{$\begin{array}{c}\text { N35 } \\
41.566 \\
\end{array}$} & \multirow{2}{*}{$\begin{array}{c}\text { E128 } \\
15.942 \\
\end{array}$} & \multirow{2}{*}{$\begin{array}{c}\text { Hydrilla } \\
\text { verticillata }\end{array}$} & Chironomidae & & 16 & & \\
\hline & & & & & & Hydrellia sp. & 1 & 1 & & \\
\hline & Munsanri (WEST) & $\begin{array}{l}3 \text { August } \\
2014\end{array}$ & $\begin{array}{c}\text { N35 } \\
51.413\end{array}$ & $\begin{array}{l}\text { E128 } \\
26.14\end{array}$ & $\begin{array}{c}\text { Hydrilla } \\
\text { verticillata } \\
\text { (US } \\
\text { monoecious?) }\end{array}$ & Nymphulinae & & & $\begin{array}{l}2 \text { (empty) } \\
\text { only) }\end{array}$ & \\
\hline \multirow[b]{2}{*}{ Country } & \multirow[b]{2}{*}{ Site } & \multirow[b]{2}{*}{ Date } & \multirow{2}{*}{\multicolumn{2}{|c|}{ GPS }} & \multirow[b]{2}{*}{ Plant Species } & \multirow[b]{2}{*}{ Herbivore } & \multicolumn{4}{|c|}{ Number/Stage } \\
\hline & & & & & & & Adults & Larvae & Pupae & Damage \\
\hline \multirow{21}{*}{$\begin{array}{l}\text { Republic of } \\
\text { Korea }\end{array}$} & Munsanri (EAST) & $\begin{array}{l}3 \text { August } \\
2014\end{array}$ & $\begin{array}{c}\text { N35 } \\
51.413\end{array}$ & $\begin{array}{l}\text { E128 } \\
26.14\end{array}$ & $\begin{array}{c}\text { Hydrilla } \\
\text { verticillata }\end{array}$ & Chironomidae & & 13 & & \\
\hline & Naktanggang & $\begin{array}{l}3 \text { August } \\
2014\end{array}$ & $\begin{array}{c}\text { N35 } \\
52.782\end{array}$ & $\begin{array}{c}\text { E128 } \\
23.702\end{array}$ & $\begin{array}{c}\text { Hydrilla } \\
\text { verticillata }\end{array}$ & Chironomidae & & 1 & & \\
\hline & \multirow{3}{*}{ Daerimdong } & \multirow{3}{*}{$\begin{array}{l}3 \text { August } \\
2014\end{array}$} & \multirow{3}{*}{$\begin{array}{c}\text { N35 } \\
52.156\end{array}$} & \multirow{3}{*}{$\begin{array}{c}\text { E128 } \\
44.742\end{array}$} & \multirow{3}{*}{$\begin{array}{c}\text { Hydrilla } \\
\text { verticillata }\end{array}$} & Acarina & 1 & & & \\
\hline & & & & & & Chironomidae & & 1 & & \\
\hline & & & & & & Nymphulinae & & 20 & & \\
\hline & Hyeongsan 1 & $\begin{array}{l}3 \text { August } \\
2014\end{array}$ & $\begin{array}{c}\text { N35 } \\
58.858\end{array}$ & $\begin{array}{c}\text { E129 } \\
15.834\end{array}$ & $\begin{array}{c}\text { Hydrilla } \\
\text { verticillata }\end{array}$ & Nil & & & & \\
\hline & Hyeongsan 2 & $\begin{array}{l}3 \text { August } \\
2014\end{array}$ & $\begin{array}{c}\text { N35 } \\
58.827 \\
\end{array}$ & $\begin{array}{c}\text { E129 } \\
15.811 \\
\end{array}$ & $\begin{array}{c}\text { Hydrilla } \\
\text { verticillata }\end{array}$ & Chironomidae & & 2 & & \\
\hline & \multirow{3}{*}{ Gunwi } & \multirow{3}{*}{$\begin{array}{l}4 \text { August } \\
2014\end{array}$} & \multirow{3}{*}{$\begin{array}{c}\mathrm{N} 36 \\
12.134\end{array}$} & \multirow{3}{*}{$\begin{array}{c}E 128 \\
33.693\end{array}$} & \multirow{3}{*}{$\begin{array}{c}\text { Hydrilla } \\
\text { verticillata }\end{array}$} & Chironomidae & & 3 & & \\
\hline & & & & & & Hydrellia sp. & & 3 & 1 & $\mathrm{X}$ \\
\hline & & & & & & Nymphulinae & & 4 & & $x$ \\
\hline & Danyang & $\begin{array}{l}4 \text { August } \\
2014\end{array}$ & $\begin{array}{c}\text { N37 } \\
01.383\end{array}$ & $\begin{array}{c}\text { E128 } \\
17.189\end{array}$ & $\begin{array}{c}\text { Hydrilla } \\
\text { verticillata }\end{array}$ & Chironomidae & & 5 & & \\
\hline & Wonju & $\begin{array}{l}4 \text { August } \\
2014\end{array}$ & $\begin{array}{c}\text { N37 } \\
25.711\end{array}$ & $\begin{array}{c}\text { E127 } \\
55.277\end{array}$ & $\begin{array}{c}\text { Hydrilla } \\
\text { verticillata }\end{array}$ & Nil & & & & \\
\hline & Wonju & $\begin{array}{l}4 \text { August } \\
2014\end{array}$ & $\begin{array}{c}\text { N37 } \\
25.711\end{array}$ & $\begin{array}{c}\text { E127 } \\
55.277\end{array}$ & $\begin{array}{l}\text { Myriophyllum } \\
\text { spicatum }\end{array}$ & Nil & & & & \\
\hline & \multirow{2}{*}{ Deokeuwonri } & \multirow{2}{*}{$\begin{array}{l}5 \text { August } \\
2014\end{array}$} & \multirow{2}{*}{$\begin{array}{c}\text { N37 } \\
51.063 \\
\end{array}$} & \multirow{2}{*}{$\begin{array}{c}\text { E127 } \\
40.239 \\
\end{array}$} & \multirow{2}{*}{$\begin{array}{c}\text { Hydrilla } \\
\text { verticillata }\end{array}$} & Chironomidae & & 81 & & $x$ \\
\hline & & & & & & Ceratopogonidae & & 1 & & \\
\hline & Deokeuwonri & $\begin{array}{l}5 \text { August } \\
2014\end{array}$ & $\begin{array}{c}\text { N37 } \\
51.063\end{array}$ & $\begin{array}{c}\text { E127 } \\
40.239\end{array}$ & $\begin{array}{l}\text { Myriophyllum } \\
\text { spicatum }\end{array}$ & Chironomidae & & 7 & & \\
\hline & \multirow{2}{*}{ Uiam-ho } & \multirow{2}{*}{$\begin{array}{l}5 \text { August } \\
2014\end{array}$} & \multirow{2}{*}{$\begin{array}{c}\text { N37 } \\
53.159\end{array}$} & E127 & Hydrilla & Chironomidae & & 3 & & \\
\hline & & & & 41.100 & verticillata & Ceratopogonidae & & 6 & & \\
\hline & Yangu City & $\begin{array}{l}5 \text { August } \\
2014 \\
\end{array}$ & $\begin{array}{c}\text { N38 } \\
05.327 \\
\end{array}$ & $\begin{array}{c}\text { E127 } \\
59.878\end{array}$ & $\begin{array}{c}\text { Hydrilla } \\
\text { verticillata }\end{array}$ & Chironomidae & & 35 & & \\
\hline & Samcheondong & 6 August & N37 & E127 & Hydrilla & Chironomidae & 1 & 18 & 2 & \\
\hline & Samineonuong & 2014 & 52.381 & 41.977 & verticillata & Trichoptera & 2 & 4 & & \\
\hline
\end{tabular}




\begin{tabular}{|c|c|c|c|c|c|c|c|c|c|c|}
\hline & Samcheondong & $\begin{array}{l}6 \text { August } \\
2014\end{array}$ & $\begin{array}{c}\text { N37 } \\
52.381\end{array}$ & $\begin{array}{c}\text { E127 } \\
41.977\end{array}$ & $\begin{array}{l}\text { Myriophyllum } \\
\text { spicatum }\end{array}$ & Nil & & & & \\
\hline & Gapyeong & $\begin{array}{l}6 \text { August } \\
2014\end{array}$ & $\begin{array}{c}\text { N37 } \\
49.640\end{array}$ & $\begin{array}{c}\mathrm{E} 127 \\
31.063\end{array}$ & $\begin{array}{c}\text { Hydrilla } \\
\text { verticillata }\end{array}$ & Chironomidae & & 3 & & \\
\hline & Daeseongri & $\begin{array}{l}6 \text { August } \\
2014\end{array}$ & $\begin{array}{c}\mathrm{N} 37 \\
43.127\end{array}$ & $\begin{array}{c}\text { E127 } \\
24.293\end{array}$ & $\begin{array}{c}\text { Hydrilla } \\
\text { verticillata }\end{array}$ & Nil & & & & $X$ (midge) \\
\hline & Songsanri & $\begin{array}{l}6 \text { August } \\
2014\end{array}$ & $\begin{array}{c}\text { N37 } \\
41.208\end{array}$ & $\begin{array}{c}\text { E127 } \\
31.272\end{array}$ & $\begin{array}{c}\text { Hydrilla } \\
\text { verticillata }\end{array}$ & Chironomidae & & 2 & & $x$ \\
\hline & & & & & & & & Num & /Stage & \\
\hline Country & Site & Date & & & Plant Species & Herbivore & Adults & Larvae & Pupae & Damage \\
\hline & Songcanri & 6 August & N37 & E127 & Myriophyllum & Chironomidae & & 7 & & \\
\hline & songsanrl & 2014 & 41.208 & 31.272 & spicatum & Trichoptera & & 1 & & \\
\hline & Munhori & 6 August & N37 & $\mathrm{E} 127$ & Hydrilla & Chironomidae & 2 & 2 & & $x$ \\
\hline & IVIuntiont & 2014 & 35.528 & 20.688 & verticillata & Nymphulinae & & 1 & & \\
\hline & Munhori & $\begin{array}{l}6 \text { August } \\
2014\end{array}$ & $\begin{array}{c}N 37 \\
35.528 \\
\end{array}$ & $\begin{array}{c}\text { E127 } \\
20.688 \\
\end{array}$ & $\begin{array}{l}\text { Myriophyllum } \\
\text { spicatum }\end{array}$ & Nil (DNA only) & & & & \\
\hline & Dogkri & 6 August & N37 & E127 & Hydrilla & Aphidae & 2 & & & \\
\hline & Logk $\Pi$ & 2014 & 31.167 & 22.535 & verticillata & Hydrellia sp. & 3 & 5 & & $x$ \\
\hline $\begin{array}{l}\text { Republic of } \\
\text { Korea }\end{array}$ & Dogkri & $\begin{array}{l}6 \text { August } \\
2014\end{array}$ & $\begin{array}{c}\text { N37 } \\
31.167\end{array}$ & $\begin{array}{c}\mathrm{E} 127 \\
22.535\end{array}$ & $\begin{array}{l}\text { Myriophyllum } \\
\text { spicatum }\end{array}$ & Nil & & & & \\
\hline & Jeongjiri Village & $\begin{array}{l}7 \text { August } \\
2014\end{array}$ & $\begin{array}{c}\text { N37 } \\
27.568\end{array}$ & $\begin{array}{c}\text { E127 } \\
18.250\end{array}$ & $\begin{array}{c}\text { Hydrilla } \\
\text { verticillata }\end{array}$ & Nil & & & & \\
\hline & Hangang & $\begin{array}{l}7 \text { August } \\
2014\end{array}$ & $\begin{array}{c}\text { N37 } \\
33.397\end{array}$ & $\begin{array}{c}\text { E127 } \\
13.376\end{array}$ & $\begin{array}{l}\text { Myriophyllum } \\
\text { spicatum }\end{array}$ & Chironomidae & & 1 & & \\
\hline & Hangana? & 7 August & N37 & E126 & Myriophyllum & Chironomidae & & 4 & & \\
\hline & Hangang 2 & 2014 & 35.323 & 50.233 & spicatum & Trichoptera & & 3 & & \\
\hline & Hangang? & 7 August & N37 & E126 & Hydrilla & Chironomidae & & 2 & & \\
\hline & Hangang 2 & 2014 & 35.323 & 50.233 & verticillata & Trichoptera & & 1 & & \\
\hline & & & & & & Chironomidae & 8 & 8 & & \\
\hline & Tonalu & 10 August & N29 & E119 & Hydrilla & Hydrellia sp. & 2 & 1 & & \\
\hline & IOnigiu & 2014 & 41.221 & 36.129 & verticillata & Trichoptera & 5 & & & \\
\hline Peoples & & & & & & Nymphulinae & & 27 & & \\
\hline China & Tonalu & 10 August & N29 & E119 & Myriophyllum & Chironomidae & 1 & 2 & & \\
\hline & Tongiu & 2014 & 41.221 & 36.129 & spicatum & Trichoptera & 5 & & & \\
\hline & Gintian & 10 August & N29 & E119 & Hydrilla & Chironomidae & & 1 & & \\
\hline & Gintian & 2014 & 37.015 & 31.959 & verticillata & Hydrellia sp. & & 1 & & $x$ \\
\hline
\end{tabular}




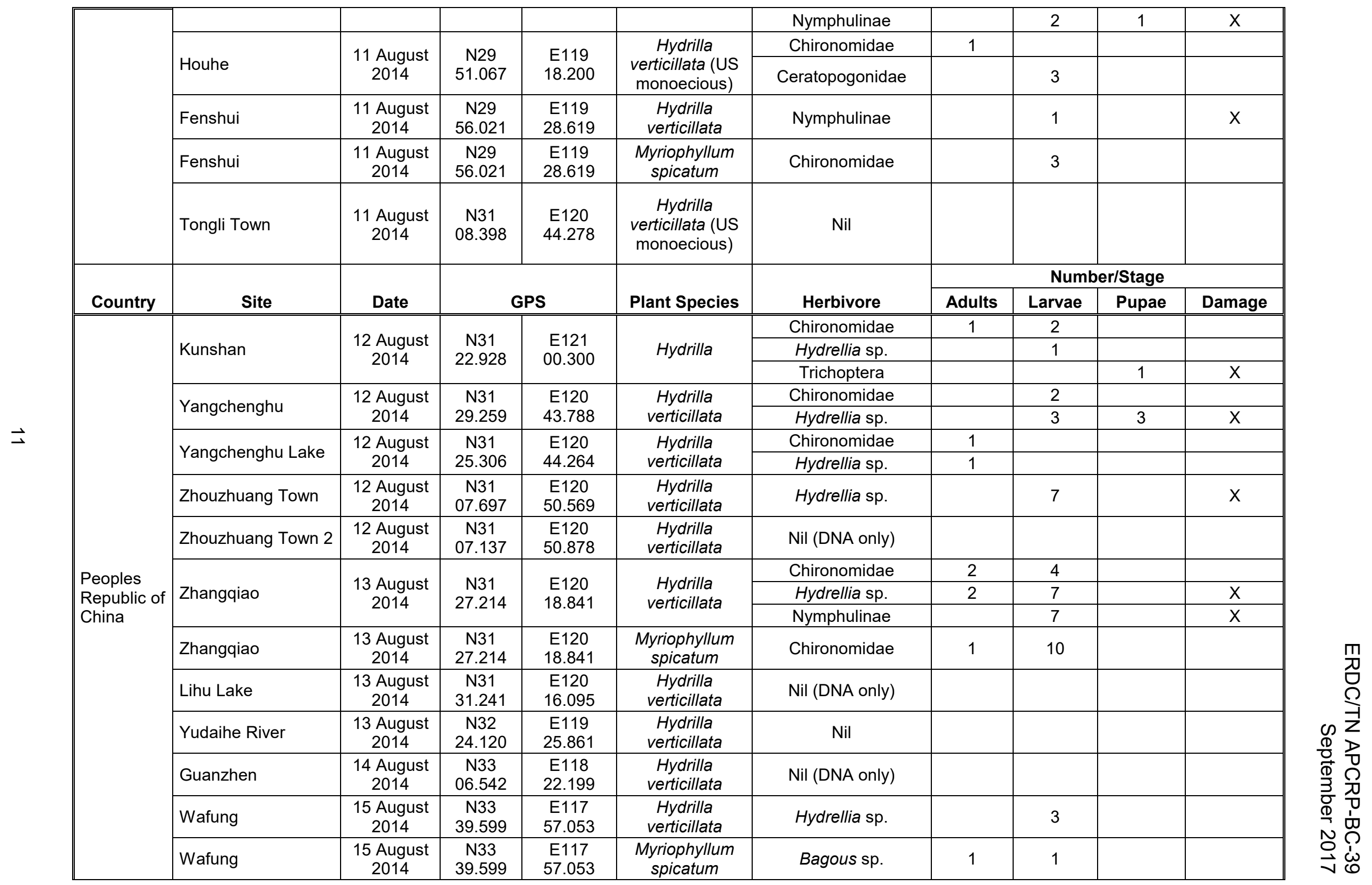




\begin{tabular}{|c|c|c|c|c|c|c|c|c|}
\hline \multirow{2}{*}{ Zhongcun Villiage } & \multirow{2}{*}{$\begin{array}{l}15 \text { August } \\
2014\end{array}$} & \multirow{2}{*}{$\begin{array}{c}\mathrm{N} 34 \\
37.243\end{array}$} & \multirow{2}{*}{$\begin{array}{l}\text { E117 } \\
22.262\end{array}$} & \multirow{2}{*}{$\begin{array}{c}\text { Hydrilla } \\
\text { verticillata }\end{array}$} & Chironomidae & & 2 & \\
\hline & & & & & Nymphulinae & & 6 & $\bar{x}$ \\
\hline \multirow{2}{*}{ Jinan } & \multirow{2}{*}{$\begin{array}{l}16 \text { August } \\
2014\end{array}$} & \multirow{2}{*}{$\begin{array}{c}N 36 \\
40.542 \\
\end{array}$} & \multirow{2}{*}{$\begin{array}{l}\text { E117 } \\
01.783 \\
\end{array}$} & \multirow{2}{*}{$\begin{array}{c}\text { Hydrilla } \\
\text { verticillata }\end{array}$} & Chironomidae & & 7 & \\
\hline & & & & & Ceratopogonidae & & 1 & \\
\hline Jinhang Channel & $\begin{array}{l}17 \text { August } \\
2014\end{array}$ & $\begin{array}{c}\text { N39 } \\
11.891 \\
\end{array}$ & $\begin{array}{c}\text { E117 } \\
08.023\end{array}$ & $\begin{array}{c}\text { Hydrilla } \\
\text { verticillata }\end{array}$ & Chironomidae & 2 & 13 & \\
\hline Jinhang Channel & $\begin{array}{l}17 \text { August } \\
2014\end{array}$ & $\begin{array}{c}\text { N39 } \\
11.891 \\
\end{array}$ & $\begin{array}{c}\text { E117 } \\
08.023 \\
\end{array}$ & $\begin{array}{c}\text { Myriophyllum } \\
\text { spicatum }\end{array}$ & Chironomidae & & 8 & \\
\hline \multirow{2}{*}{ Langfang } & \multirow{2}{*}{$\begin{array}{l}17 \text { August } \\
2014\end{array}$} & \multirow{2}{*}{$\begin{array}{c}\text { N39 } \\
35.034\end{array}$} & \multirow{2}{*}{$\begin{array}{c}\text { E116 } \\
45.821\end{array}$} & \multirow{2}{*}{$\begin{array}{c}\text { Hydrilla } \\
\text { verticillata }\end{array}$} & Chironomidae & 4 & $\sim 200$ & $\mathrm{x}$ \\
\hline & & & & & Hydrellia sp. & & 15 & $\mathrm{x}$ \\
\hline $\begin{array}{l}\text { Royal Bamboo Park } \\
2\end{array}$ & $\begin{array}{l}18 \text { August } \\
2014\end{array}$ & $\begin{array}{c}\text { N39 } \\
56.433\end{array}$ & $\begin{array}{c}\text { E116 } \\
18.907\end{array}$ & $\begin{array}{l}\text { Myriophyllum } \\
\text { spicatum }\end{array}$ & Nil (DNA only) & & & \\
\hline August 1st Park & $\begin{array}{l}18 \text { August } \\
2014\end{array}$ & $\begin{array}{c}\mathrm{N} 39 \\
55.021\end{array}$ & $\begin{array}{c}\text { E116 } \\
18.740\end{array}$ & $\begin{array}{l}\text { Myriophyllum } \\
\text { spicatum }\end{array}$ & Nil (DNA Only) & & & \\
\hline
\end{tabular}


Hydrilla. The most common herbivore on hydrilla collected at all sites were Chironomidae, with adults or larvae collected from $61 \%$ and $59 \%$ of the RK and the PRC hydrilla sites, respectively (Table 3). The larvae of the majority of midges appeared to feed on apical meristems, resulting in reduced elongation and increased branching near stem apices. More significantly, hydrilla from Langfang, PRC was heavily infested by defoliating chironomids, with more than 200 larvae and four adults being extracted from a hydrilla sample processed in the Berlese funnel (Figure 3).

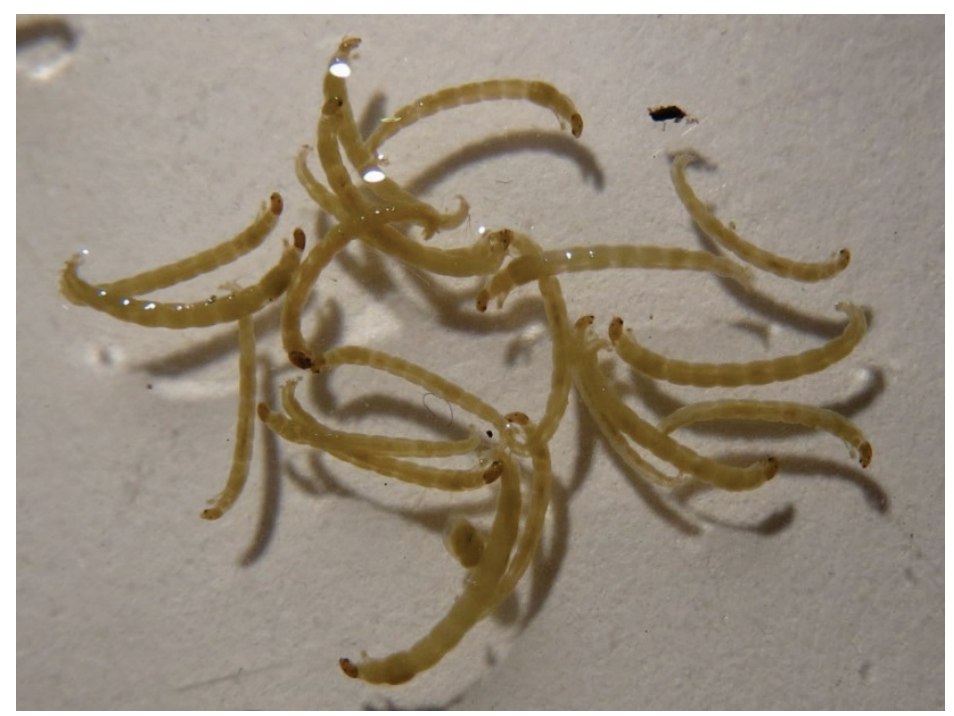

Figure 3. Chironomidae larvae had defoliated hydrilla at Langfang, PRC.

Plants at Langfang were defoliated from the larvae that fed on leaves. The site was heavily polluted with rubbish (Figure 4) with poor water quality; high $\mathrm{pH}$ (10.3), total dissolved solids (>1200 ppm) and salinity (798 ppm). It is unclear whether poor water quality or eutrophication contributed to the large midge populations. However, growth of some midges can be enhanced by such conditions or parasitism/predation could be reduced; some predatory invertebrates are sensitive to high levels of pollutants (Stuijfzand et al. 2000; Bulánková 1997). Once identified, further investigations will be conducted on this species (adult chironomids collected from the field and reared in captivity during the current survey are being identified by Dr. Peter Cranston, a retired dipterist and Chironomidae specialist in Australia).

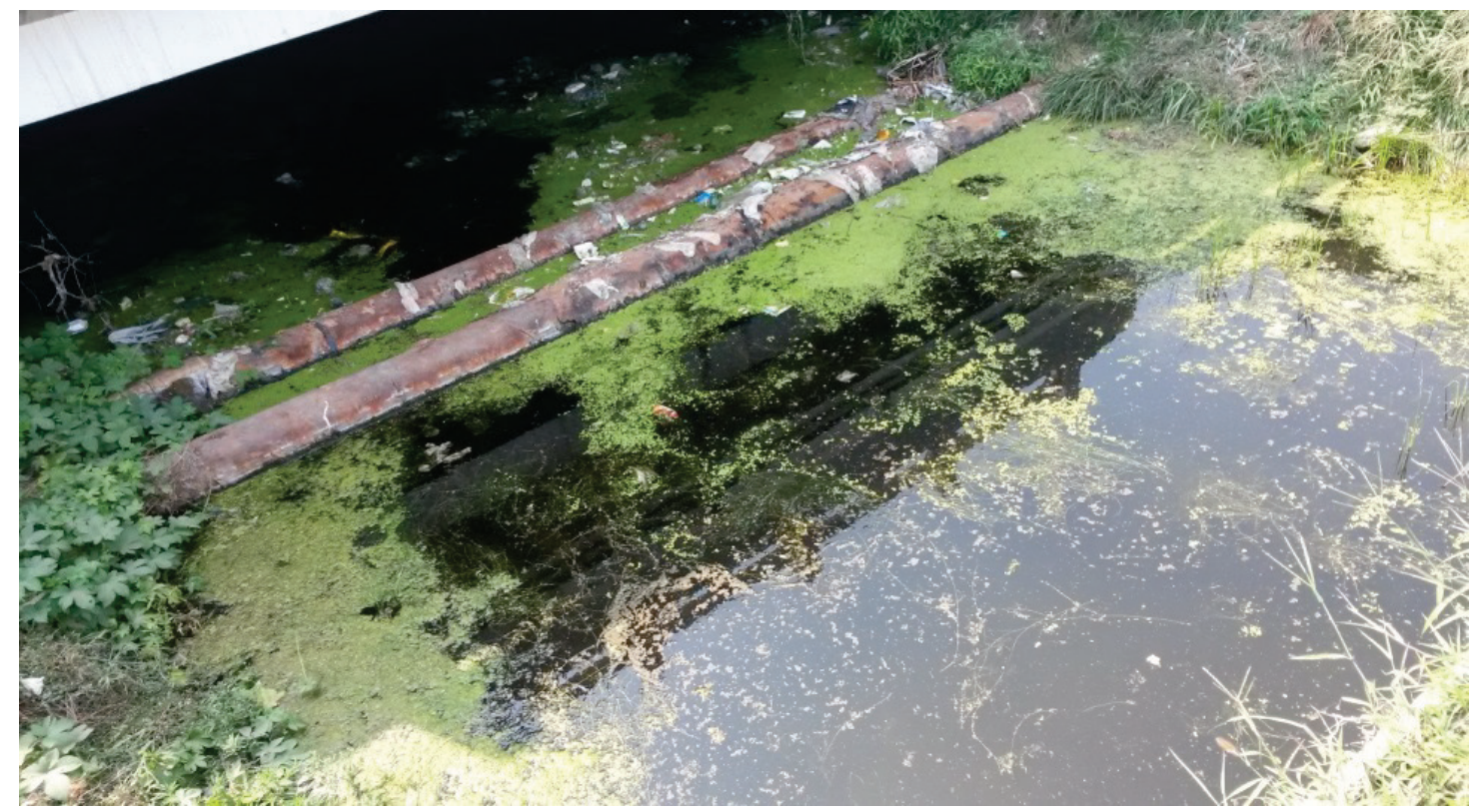

Figure 4. Hydrilla heavily infested by leaf-feeding Chironomidae at a highly polluted site at Langfang, PRC. 
Leaf-mining Hydrellia sp. flies were collected from nine sites (20\%) in the RK and nine sites (47\%) in the PRC. A total of four adults, one pupa and 23 larvae were collected from RK while five adults, three pupae and 38 larvae were collected from the PRC. Leaf-mining damage was frequently observed when searching plant material with a hand lens, though the impact was usually minimal given the low populations of larvae at a given site. Micro-hymenoptera were extracted from the Berlese funnels on several occasions indicating that field populations may be regulated by parasites. Adult flies were seen flying above the water surface or resting on emergent material. Adults will be identified by Dr. John Deeming from the National Museum of Wales who is currently reviewing the genus Hydrellia.

Berlese funnel processing and hand searching of hydrilla samples extracted 76 aquatic moth larvae, four pupae and one adult from 13 field sites in the RK. An adult moth from Hotan was identified by Dr. Shen-Horn Yen from the National Sun Yat-sen University in Taiwan as Parapoynx vittalis, as were two specimens reared from immatures collected at the Gum River and Yeonpori (West). This species has been collected from hydrilla elsewhere in Asia and other suspected hosts in the literature include records from Poaceae, Potamogetonaceae and Cabombaceae in Japan and from rice in China ${ }^{1}$. Forty-three aquatic moth larvae and one pupa were collected from five sites in the PRC, but no adults were reared. Like Hydrellia, moth damage was readily observed at many sites by inspecting hydrilla using a hand lens, though the overall impact to plants was minimal.

No Bagous weevils were collected on hydrilla in either the RK or the PRC during 2014. Sites where Bagous sp. 2 and B. rufipennis are reliably collected in Hubei, PRC were adversely affected by flooding. A colony of B. rufipennis is still being maintained at the Austrailia Biological Control Lab (ABCL) for quarantine in Brisbane but fails to thrive, probably due to long-term quarantine confinement. In host range testing, this weevil fed on hydrilla and two other hosts but, testing of a full array of species has yet to be completed. New stock will be imported from China to boost the vigor of the B. rufipennis colony so that testing can be finalized. Bagous sp. 2 has yet to be colonized and tested. It is believed that this species pupates underwater and does not require dry periods or shoreline access.

Adults and larval Elmidae (Coleoptera) were collected in the RK at three sites but not in the PRC, though specimens were collected during previous surveys in the PRC in $2013^{2}$. As in China, specimens from the RK did not appear to damage hydrilla and likely feed on detritus and algae as is common for this family.

Caddisflies (Trichoptera) were also extracted from Berlese funnels from five sites in the RK (three adults, nine larvae) and two sites in China (five adults, one pupae). The case-forming larvae likely fed on plant material but we could not determine if adults extracted from samples fed on hydrilla in the larval stage. Other possible herbivores included one aquatic mite and two aphids from the RK.

Monoecious hydrilla could not be morphologically identified at any of the sites visited in the RK or the PRC. Male and female flowers were always collected on separate plants or no flowers were

\footnotetext{
${ }^{1}$ Shen-Horn Yen, National Sun Yat-sen Univ., Dept. Biol. Sci., Kaohsiung Taiwan.

${ }^{2}$ Harms, N. E., M. Purcell, J. Zhang, M. J. Grodowitz and J. Ding. In Prep. Surveys for Biological Control Agents of Hydrilla verticillata in the People's Republic of China during 2013.
} 
found at all. However, these results are not conclusive in determining if monoecious plants were present or collected, especially at sites where the hydrilla contained no flowers. A concurrent study to genetically characterize samples will determine biotype (monoecious or dioecious) and genetic similarity to U.S. hydrilla plants. Preliminary information from this study confirms that four sites (two in the PRC and two in the RK) contained hydrilla that is the exact match for the U.S. monoecious biotype ${ }^{1}$.

EWM. Similar to hydrilla, the most common herbivore extracted from EWM samples processed in Berlese funnels were chironomids. In the RK, 65 larvae were collected from seven sites, while in the PRC, two adults and 23 larvae were collected from four sites. Unlike hydrilla, the effects of larval damage were not as obvious on infested plants. Five Trichoptera larvae and five adults were collected from the RK and the PRC respectively. A Bagous adult (Figure 5) was collected from EWM at Wafung, PRC. Additional surveys in September, 2014 collected larvae and

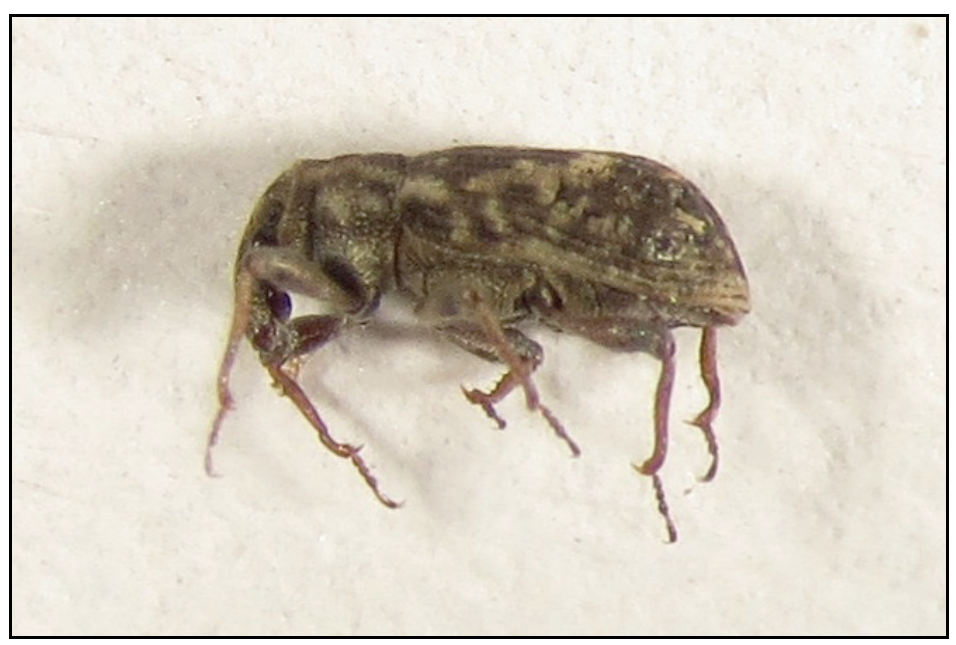

Figure 5. Bagous sp. collected from Eurasian watermilfoil at Wafung, PRC. adults from EWM but also three larvae from hydrilla, though these were thought to be contaminants. Adults were collected from the field and reared from larvae on EWM but could not be colonized in laboratory studies. The adults were identified by Dr. Charles O'Brien in 2015 as B. myriophylli. This species completes its life cycle underwater and was imported into quarantine in the U.S. in 1992, but host range testing indicated that it would complete development on four other Myriophyllum sp. and the close relative Proserpinaca palustris L. (Bennett and Buckingham 1999). Additionally, adults and immatures were collected from the field in the PRC attacking both $M$. spicatum and M. verticillatum, the latter being native to North America (Bennett and Buckingham 1999).

FUTURE DIRECTIONS. Results from a parallel study genetically characterizing hydrilla samples collected from the RK and the PRC in 2014 will guide future surveys. Sites where monoecious hydrilla matches the U.S. biotype and new sites located in the surrounding areas will be targeted in greater detail over the entire growing season. Herbivores identified from these areas will be imported into quarantine facilities in Brisbane, Australia for colonization and host range evaluations. In addition, observations will be made on plant growth characteristics and herbivore damage.

As potential biological control agents, the Chironomidae (Figure 3) defoliating hydrilla at Langfang, PRC, Hydrellia flies collected from monoecious hydrilla in the RK and the PRC, and the Bagous sp. collected from EWM at Wafung, PRC are priorities. Chironomids have received little historical

\footnotetext{
${ }^{1}$ Dean Williams, Texas Christian University, Dept. of Biology, Fort Worth, TX, U.S.A.
} 
attention as hydrilla biological control agents because it was assumed that tip damage has insufficient impact on plant growth. However, as the discovery of new potential agents is indicating, there is renewed interest in midges. In addition to identifying leaf-feeding midges collected at Langfang in 2014, historic collections from 1996 - present will be examined and identified to determine which species have been previously associated with hydrilla and their potential geographic ranges.

Bagous sp. 2 from hydrilla in Hubei Province, PRC will be collected for colonization, specificity testing, and to assess its ability to complete development underwater. No further studies are planned on B. myriophylli collected from EWM at Wafung, PRC given its lack of specificity in field and quarantine studies (Bennett and Buckingham 1999).

ACKNOWLEDGEMENTS: This research was supported by the U.S. Army Aquatic Plant Control Research Program (APCRP), under the management of Dr. Linda Nelson. Permission was granted by the Chief of Engineers to publish this information. The authors would also thank Gary Dick and Judy Shearer for their review of this manuscript.

ADDITIONAL INFORMATION: Contact the author, Nathan E. Harms, (601) 634-2976, Nathan.E.Harms@usace.army.mil or the Program Manager, Linda Nelson, (601) 634-2656, Linda.S.Nelson@usace.army.mil. This technical note should be cited as follows:

Purcell, M., N. E. Harms, M. J. Grodowitz, J. Zhang, M. H. Kim, K. J. Cho, Y. J. Oh, S. -Hee Hong and J. Ding. 2017. Surveys for Biological Control Agents of Hydrilla verticillata and Myriophyllum spicatum in the Republic of Korea and the People's Republic of China in 2014. ERDC/TN APCRP-BC-39. Vicksburg, MS: U.S. Army Engineer Research and Development Center.

\section{REFERENCES}

Balciunas, J. K., M. J. Grodowitz, A. F. Cofrancesco, and J. F. Shearer. 2002. Hydrilla. In Biological Control of Invasive Plants in the Eastern United States, eds R. Van Driesche, B. Blossey, M. Hoddle, S. Lyon and R. Reardon, 184-195. USDA Forest Service Publication FHTET-2002-04.

Bennett, C. A., and G. R. Buckingham. 1999. Biological control of hydrilla and Eurasian watermilfoil-insect quarantine research. In: Florida's Garden of Good and Evil: In Proceedings of the 1998 Joint Symposium of the Florida Exotic Pest Plant Council and the Florida Native Plant Society, ed. D. T. Jones, and B. W. Gamble, 363-369. West Palm Beach, FL: South Florida Water Management District.

Benoit, L. 2011. Cryptic speciation, genetic diversity and herbicide resistance in the invasive aquatic plant Hydrilla verticillata (L.f.) Royle (Hydrocharitaceae). PhD diss., Univ. of Connecticut.

Buckingham, G. R. 2004. In: Biological Control of Invasive Plants in the United States, ed. E. M. Coombs, J. K. Clark, G. L. Piper, and A. F. Cofrancesco, 169-173. Corvallis, OR: Oregon State University Press.

Buckingham, G. R., and M. J. Grodowitz. 2004. Hydrilla. In: Biological Control of Invasive Plants in the United States, ed. E. M. Coombs, J. K. Clark, G. L. Piper, and A. F. Cofrancesco, 184-195. Corvallis, OR: Oregon State University Press.

Bulánková, E. 1997. Dragonflies (Odonata) as bioindicators of environment quality. Biologia 52(2):177-180.

Couch, R., and E. Nelson. 1985. Myriophyllum spicatum in North America. In Aquatic Plant Management Society ed. W. J. Anderson. First International Symposium on Watermilfoil and Related Haloragaceae Species. 23-24 July 1985, Vancouver, BC. 
Dray, F. A., and T. D. Center. 1996. Reproduction and development of the biocontrol agent Hydrilla pakistanae (Diptera: Ephydridae) on monoecious hydrilla. Biological Control 7(3):275-280. http://dx.doi.org/10.1006/bcon.1996.0094.

Eiswerth, M. E., Donaldson, S. G. and W. S. Johnson. 2000. Potential environmental impacts and economic damage of Eurasian watermilfoil (Myriophyllum spicatum) in western Nevada and northeastern California. Weed Technology 14(3):511-518. http://www.jstor.org/stable/3988902.

Grodowitz, M. J., M. Smart, R. D. Doyle, C. S. Owens, R. Bare, C. Snell, J. Freedman, and H. Jones. 2004. Hydrellia pakistanae and $\mathrm{H}$. balciunasi, insect biological control agents of hydrilla: boon or bust? In Proceedings of the XI International Symposium on Biological Control of Weeds, ed. J. M. Cullen, D. T. Briese, D. J. Kriticos, W. M. Lonsdale, L. Morin, and J. K. Scott, 529-538. Canberra, Australia: CSIRO.

Grodowitz M. J., J. Nachtrieb, N. E. Harms, and J. Freedman. 2010. Suitability of using introduced Hydrellia spp. for management of monoecious Hydrilla verticillata (L.f) Royle. APCRP Technical Notes Collection, ERDC/TN APCRPBC-17. Vicksburg, MS: U.S. Army Engineer Research and Development Center.

Gross, E. M., R. L. Johnson, and N. G. Hairston Jr. 2001. Experimental evidence for changes in submersed macrophyte species composition caused by the herbivore Acentria ephemerella (Lepidoptera). Oecologia 127(1):105-114. http://www.jstor.org/stable/4222904.

Harms, N. E., and M. J. Grodowitz. 2011. Overwintering biology of Hydrellia pakistanae, a biological control agent of hydrilla. Journal of Aquatic Plant Management 49:114-117.

Harms, N. E., D. A. Williams, and M. J. Grodowitz. 2014. Genetic relationships among invasive Hydrilla (Hydrilla verticillata L.f. Royle) biotypes in the U.S. and their implications for management. APCRP Technical Notes Collection. ERDC/TN APCRP-BC-32. Vicksburg, MS: U.S. Army Engineer Research and Development Center.

Jacono, C. C., M.M. Richerson, V. Howard Morgan, and I. A. Pfingsten. 2017. Hydrilla verticillata [monoecious] (L. f.) Royle: U.S. Geological Survey, Nonindigenous Aquatic Species Database, Gainesville: FL, https://nas.er.usgs.gov/queries/FactSheet.aspx?SpeciesID=2943, Revision Date: 10/27/2015, Access Date: 9/7/2017

Johnson, R. L., E. M. Gross, and N. G. Hairston Jr. 1997. Decline in the invasive submerged macrophyte Myriophyllum spicatum (Haloragaceae) associated with herbivory by larvae of Acentria ephemerella (Lepidoptera). Aquatic Ecology 31:273-282. doi:10.1023/A:1009960131857.

Johnson, R. L., and B. Blossey. 2002. Eurasian Watermilfoil. In: Biological Control of Invasive Plants in the Eastern United States., ed. R. Van Driesche, B. Blossey, M. Hoddle, S. Lyon and R. Reardon, 79-90. USDA Forest Service Publication FHTET-2002-04, 413.

Madeira, P. T., C. C. Jacono, and T. K. Van. 2000. Monitoring hydrilla using two RAPD procedures and the nonindigenous database. Journal of Aquatic Plant Management 38:33-40.

Madeira, P. T., J. A. Coetzee, T. D. Center, E. E. White, and P. W. Tipping. 2007. The origin of Hydrilla verticillata recently discovered at a South African dam. Aquatic Botany 87(2):176-180. doi: http://dx.doi.org/10.1016/j.aquabot.2007.04.008.

Painter, D. S., and K. J. McCabe. 1988. Investigation into the disappearance of Eurasian watermilfoil from the Kawartha Lakes. Journal of Aquatic Plant Management 26:3-12.

Schmitz D. C., B. V. Nelson, L. E. Nail, and J. D. Schardt. 1991. Exotic aquatic plants in Florida: A historical perspective and review of the present aquatic plant regulation program. In: Proceedings of the Symposium on Exotic Pest Plants: November 2-4, 1988, University of Miami, Rosensteil School of Marine and Atmospheric Science, Miami, FL, ed. T. D. Center, R. F. Doren, R. L. Hofstetter, R. L. Myyers, and L. D. Whiteaker, 303-323. Washington, DC: United States Department of the Interior, National Park Service Document.

Sheppard, A. C. 1945. A new record for Canada (Lepidoptera). The Canadian. Entomologist 77(3):55. doi: https://doi.org/10.4039/Ent7755-3.

Smith, C. S., and J. W. Barko. 1990. Ecology of Eurasian watermilfoil. Journal of Aquatic Plant Management 28:55-64. 
Steward, K. K., T. K. Van, C. Carter, and A. H. Pieterse. 1984. Hydrilla invades Washington, D.C. and the Potomac. American Journal of Botany 71:162-163. doi: 10.2307/24433637.

Stuijfzand, S. C., M. Helms, M. H. S. Kraak, and W. Admiraal. 2000. Interacting effects of toxicants and organic matter on the midge Chironomus riparius in polluted river water. Ecotoxicology and Environmental Safety 46(3):351-356. doi: 10.1006/eesa.2000.1918.

U.S. Congress, Office of Technology Assessment 1993. Harmful Non-Indigenous Species in the United States. OTA-F-565. Washington, D.C.

NOTE: The contents of this technical note are not to be used for advertising, publication or promotional purposes.

Citation of trade names does not constitute an official endorsement or approval of the use of such products. 